\title{
Fatores Associados ao Hábito de Assistir TV em Excesso Entre Adolescentes
}

\section{Factors Associated With Excessive Television Watching Among Adolescents}

\author{
Paula Dal Bó Campagnolo’ \\ Márcia Regina Vitolo \\ Cíntia Mendes Gama
}

1. Universidade Federal de Ciências da Saúde de Porto Alegre (UFCSPA)

\section{Endereço para correspondência: \\ Rua Santa Rita, 285/202 \\ CEP 90220-220 \\ Porto Alegre - RS - Brazil \\ Email: pcampagnolo@hotmail.com}

Submetido em 14/09/2007 Versão final recebida em 23/10/2007 Aceito em 03/01/2008

\begin{abstract}
RESUMO
Objetivo: Avaliar a freqüência com que os adolescentes assistem televisão na região Sul do Brasil e quais fatores estão associados a esse comportamento. Métodos: Estudo transversal, com 722 adolescentes entre 10 e 19 anos. Foi calculado a média de horas diárias que os adolescentes assistiam televisão. Foi realizada análise de regressão logística para analisar possível associação entre fatores sociodemográfico, antropométricos, atividade física, sexo e idade, com o hábito de assistir mais do que 4 horas diárias de televisão. Resultados: Entre os adolescentes estudados, 41,1\% assistiam mais do 4 horas de TV por dia. A prevalência de adolescentes que assistiam > 4h de TV foi maior entre aqueles com menos de 16 anos. Ser filho de mãe com escolaridade maior do que 8 anos (OR 1,9; IC 1,25-2,89) e possuir circunferência da cintura maior do que o percentil 80 (OR 2,77; IC 1,33-5,77) foi positivamente associado ao hábito de assistir televisão mais do que 4 horas diárias. Conclusões: Maior escolaridade materna e circunferência da cintura maior do que o percentil 80 estão associados ao comportamento de assistir TV em excesso entre os adolescentes. Esses achados devem ser utilizados para direcionar a elaboração de programas de saúde pública, especialmente por meio das escolas, enfocando adolescentes com faixa etária entre 10 e 15 anos, assim como devem servir de alerta para os profissionais de saúde e educadores.
\end{abstract}

Palavras-chave: televisão, adolescente, obesidade.

\begin{abstract}
Objective: to evaluate the frequency adolescents watch TV in southern Brazil and which factors are associated with this behavior. Methods: This was a cross-sectional study. The sample consisted of 722 adolescents between 10 and 19 years of age. Logistic regression analysis was used to verify association between sociodemographic and anthropometric factors, physical activity, age and sex with the adolescents who watched $T V$ for more than four daily hours. Results: Forty-one percent of the adolescents watched more than four daily hours of TV. The prevalence of adolescents who excessively watched TV was higher among those under 16 yr of age (PR 1.92; Cl 1.33-2.78). Having a mother with higher educational background (PR 1.51; Cl 1.08-2.11), and having waist circumference above 80 percentile (PR 1.63; $\mathrm{Cl} 1.09-2.44)$ were positively associated with the habit of watching more than 4 hours/day of television. Conclusions: Higher mother educational background and higher waist circumference are associated with excessive television watching. These results can be useful to create health programs at schools, focusing adolescents between 19 and 15 years old.
\end{abstract}

Keywords: television, adolescent, obesity.

\section{INTRODUÇÃO}

A prevalência de excesso de peso entre os adolescentes brasileiros aumentou de 3,7\% na década de 70 para 12,6\% na década de 90, similar a média de aumento anual dos Estados Unidos ${ }^{(1)}$. Este quadro é conseqüência do processo de transição nutricional que o Brasil vem passando nas últimas décadas(2), acompanhado de mudanças no hábito alimentar da população, o qual passou a ser caracterizado pelo aumento no consumo de lipídio, gordura saturada e açúcar, redução do consumo de leguminosas, verduras, legumes e frutas ${ }^{(3)}$.

A redução da atividade física de rotina e o aumento de atividades sedentárias têm papel importante na etiologia da obesidade na infância e adolescência ${ }^{(4)}$. A associação entre obesidade e tempo gasto assistindo televisão (TV) já foi demonstrado por vários estudos ${ }^{(5-10)}$, sendo a probabilidade de ser obeso três vezes maior em adolescentes que assistem TV por mais de 5 horas/dia quando comparado com aqueles que têm essa prática por $\leq 2$ horas/dia ${ }^{(11)}$. Tal associação possui diversas explicações como baixo nível de atividade física(5), hábito de comer à frente da televisão e influência das propagandas nas escolhas alimentares dos adolescentes por alimentos de alta densidade energética ${ }^{(12,13)}$.

O US Department of Health and Human Services elaborou o Programa Health People $2010^{(14)}$ o qual propõe objetivos a serem alcançados no âmbito da Saúde Pública. Entre eles, está o de aumentar para 75\% a prevalência de crianças e adolescentes que assistem 2 horas ou menos de TV por dia, salientando a relevância do estudo dessa prática. Porém, poucos estudos explicam quais os fatores que predispõem o hábito de assistir TV em excesso. Essa resposta é relevante para direcionar a elaboração de programas de saúde pública, assim como servir de alerta para os profissionais de saúde e educadores. Diante da inexistência de estudos com essas características, o objetivo desse estudo foi avaliar a freqüência com que os adolescentes assistem à televisão 
em amostra representativa de uma cidade do Rio Grande do Sul e analisar a associação dessa prática com fatores sociodemográficos e antropométricos.

\section{MATERIAIS E MÉTODOS}

Essas análises fazem parte de um estudo maior de delineamento transversal intitulado "Vigilância Nutricional: Hábito Alimentar, Antropometria e Exame Bioquímico em adolescentes moradores da cidade de São Leopoldo, RS", realizado entre os anos de 2002 e 2003, cujo objetivo principal foi estudar o excesso de peso entre os adolescentes.

O tamanho da amostra foi calculado considerando prevalência de excesso de peso de 18\%, nível de confiança de $95 \%$ e poder estatístico de $80 \%$, adicionado de $10 \%$ para as possíveis perdas, o que determinou número amostral de 810 adolescentes. Os critérios utilizados para exclusão foram: adolescentes gestantes ou nutrizes ou mães, adolescentes com deficiência física ou mental; adolescente com diabetes, hipo ou hipertireoidismo.

O processo amostral se deu por conglomerados, em 3 estágios de seleção: sorteio sistemático de 40 setores censitários, posterior sorteio aleatório das quadras e esquinas pelas quais se iniciaria a coleta e finalmente amostragem sistemática (1 em cada 3) de domicílios. Todos os indivíduos entre 10 e 19 anos morando nestes domicílios foram identificados e convidados a participar do estudo. As entrevistas foram agendadas em turnos diurnos, em todos os dias da semana, exceto aos domingos e segundas-feiras, conforme a disponibilidade do adolescente. A duração da entrevista foi de uma hora e meia, sendo o adolescente informado, previamente, desse tempo a ser despendido.

Foi elaborado questionário pré-codificado abordando fatores socioeconômicos, familiares e antropométricos. Os dados foram coletados por estudantes de graduação, especificamente treinados para as entrevistas e mensurações, além do recebimento de manual. Foi realizado estudo piloto com 60 adolescentes em setores não sorteados no processo de amostragem.

Os dados para caracterização social da população estudada foram obtidos por meio de informações sobre a escolaridade da mãe, categorizada em até 8 anos ou maior que 8 anos de estudo e se a mãe trabalhava fora de casa. Os adolescentes foram questionados quanto a percepção pessoal sobre o seu corpo e foram categorizados em satisfeitos ou insatisfeitos com seu corpo.

Os adolescentes responderam a um recordatório de atividades físicas de 7 dias ${ }^{(15)}$, no qual relataram todas as suas atividades diárias (horas de sono, sentado assistindo televisão, sentado estudando, arrumando a casa...), de lazer (passeando com amigos, andando de bicicleta, brincadeiras de rua, jogos de bola, dança...) e exercícios físicos regulares (escolinha de futebol, balé, vôlei, natação...) em horas e minutos durante os 7 dias da semana. A partir desse questionário foi calculada a média de horas diárias que o adolescente passava sentado à frente da televisão e se praticava ou não exercício físico regular, não incluindo as aulas de educação física na escola. Exercício físico regular foi considerado quando o adolescente praticava alguma atividade física programada e periodicamente, como natação, escolinha de esportes, academia e outras. Acredita-se que o método utilizado para obter as horas diárias de TV e a prática de exercício físico minimizou a possibilidade de superestimação da prática de atividade física e subestimação dos comportamentos sedentários, pois se pediu aos participantes que relatassem todas as suas atividades diárias e teve-se o cuidado de não expor aos entrevistadores e aos entrevistados a hipótese investigada, o que poderia induzir a resposta caracterizando viés de aferição.

A medida da circunferência da cintura foi obtida na menor circunferência entre o gradil costal e a cicatriz umbilical. O percentil acima de 80 para circunferência da cintura proposto por Taylor et al(16) representou o excesso de gordura localizada na região abdominal.

O teste t de Student foi utilizado para comparar a média de horas diárias que os adolescentes assistiam entre as variáveis dicotômicas. As estimativas fornecidas pelas análises bivariada e multivariada foram expressas como Odds Ratio (OR) e intervalo de confiança de 95\% (IC95\%). Para análise de regressão logística foi elaborado modelo hierárquico. As variáveis foram incluídas no modelo quando alcançaram nível de significância de $20 \%$ e a associação foi considerada significante quando atingiram 5\%. No primeiro nível foram colocadas as variáveis sexo e idade; no segundo nível as variáveis escolaridade materna e ser filho único; no terceiro nível a variável circunferência da cintura e exercício físico regular. Como desfecho considerou-se o hábito de assistir televisão em excesso. O número de horas diárias que os adolescentes assistiam à televisão foi dividido em quartis e foi considerado o último quartil como ponto de corte do desfecho (> 4 horas por dia).

Este trabalho foi aprovado pelo Comitê de Ética em Pesquisa da Universidade Federal do Rio Grande do Sul. Todos os adolescentes foram previamente informados do teor do estudo e, juntamente com seus pais, foram submetidos a um documento para formalização do consentimento de participação na pesquisa. No momento de diagnóstico de inadequação do estado nutricional, os adolescentes e seus pais foram devidamente encaminhados ao Serviço de Saúde.

\section{RESULTADOS}

Do total de 810 adolescentes identificados nos domicílios e elegíveis para o estudo, houve recusa por parte dos pais, ou do próprio adolescente, representada por $8,6 \%(n=70)$ dos convidados a participar, $1,8 \%(n=15)$ não foram encontrados nos domicílios para a realização da entrevista após três tentativas e 0,3\% ( $n=3)$ mudaram de endereço. As perdas foram maiores para o sexo masculino, considerando que das recusas, 85,7\% corresponderam a esse sexo.

Foram estudados, então, 722 adolescentes, sendo 40,6\% ( $n=293$ ) do sexo masculino e $59,4 \%(n=429)$ do sexo feminino. A média de idade foi de 14,4 2,73 , sendo que $70,6 \%$ da amostra tinha entre 16 e 19 anos. Quanto a escolaridade materna, 28\% tinham 4 anos ou menos de estudo e 34,4\% estudaram por mais de 8 anos. Praticavam exercício físico regular 30\% dos adolescentes e 18,8\% estava acima do percentil 80 para circunferência da cintura.

Observou-se que 75\% dos adolescentes assistiam mais do que 2 horas e $41 \%$ mais do que 4 horas por dia de televisão. A tabela 1 mostra a média de horas diárias que os adolescentes assistiam TV em relação ao sexo, idade, escolaridade materna, circunferência da cintura e prática de exercício físico regular. A média de horas diárias de televisão foi significativamente maior entre os adolescentes com menos de 16 anos $(p<0,001)$ e cujas mães estudaram mais do que 8 anos $(p=0,009)$.

Na tabela 2 está exposta a análise bruta e ajustada das variáveis em estudo. Houve associação positiva entre o hábito de assistir televisão mais do que 4 horas por dia e ter menos do que 16 anos (OR 1,92: IC 1,33-2,78) e apresentar circunferência da cintura maior do que o percentil 80 (OR 1,63; IC 1,09-2,44). Os Adolescentes cujas mães tinham mais do que 8 anos de escolaridade apresentaram maior frequência do hábito de assistir TV mais do que 4 horas por dia (OR 1,51; IC 1,08-2,11). A variável filho único perdeu associação com o desfecho após a análise multivariada.

\section{DISCUSSÃO}

Observou-se que $41 \%$ dos adolescentes entrevistados tinham o hábito de assistirTV mais do que 4 horas diárias e 75\% assistiam mais do que 2 horas por dia. Ao considerar que o objetivo do O US Department 
Tabela 1. Média e desvio padrão do número de horas diárias de televisão de acordo com as variáveis categóricas entre adolescentes, São Leopoldo (RS), Brasil, 2002-2003.

\begin{tabular}{|c|c|c|}
\hline & Média \pm DP & $p^{*}$ \\
\hline Meninos & $3,51 \pm 2,21$ & 0,574 \\
\hline Meninas & $3,60 \pm 2,03$ & \\
\hline 16 a 19 anos de idade & $3,09 \pm 2,06$ & 0,000 \\
\hline 10 a 15 anos de idade & $3,77 \pm 2,09$ & \\
\hline Mãe com até 8 anos de estudo & $3,41 \pm 2,13$ & 0,009 \\
\hline Mãe com > 8 anos de estudo & $3,85 \pm 2,04$ & \\
\hline Circunferência da cintura $<$ percentil 80 & $3,50 \pm 2,08$ & 0,071 \\
\hline Circunferência da cintura $\geq$ percentil 80 & $3,87 \pm 2,16$ & \\
\hline Faz exercício físico regular & $3,52 \pm 1,95$ & 0,700 \\
\hline Não faz exercício físico regular & $3,59 \pm 2,16$ & \\
\hline
\end{tabular}

DP: desvio padrão

* teste t de Student

of Health and Human Services é que $75 \%$ das crianças e adolescentes assistam 2 horas ou menos de TV por dia, que é o ponto de corte recomendado pela American Academy of Pediatrics ${ }^{(17)}$, podemos aludir que alcançar essa meta é um enorme desafio em nosso meio. Outros estudos de base populacional realizados no Brasil também mostram condições muito aquém das esperadas. A prevalência de escolares da cidade de São Paulo que assistiam 4 horas ou mais de TV por dia foi de $27,8 \%{ }^{(18)}$. Na cidade de Pelotas 33,6\% dos adolescentes assistiam 5 horas ou mais de televisão por dia ${ }^{(19)}$. Esses estudos avaliaram a prevalência de adolescentes que assistiam horas excessivas de televisão, porém não mostram dados dos fatores que estão associados a essa prática.

Mudar esse comportamento é prioridade no âmbito da Saúde Pública e quanto mais precocemente houver intervenção, mais benefícios para a saúde da criança e do adolescente. Além disso, estudo de coorte publicado recentemente demonstrou que excesso de TV durante a infância está associado a menor escolaridade aos 26 anos de idade ${ }^{(20)}$.

O comportamento de assistir mais do que quatro horas de TV por dia, nesse estudo, foi associado a adolescentes na faixa etária menor que 16 anos, maior escolaridade materna e adolescentes com circunferência da cintura $\geq$ percentil 80 . 0 percentil da circunferência da cintura parece ser um indicador eficaz em demonstrar a associação entre horas gasta assistindo TV e elevada adiposidade corporal, além de ser uma medida de fácil obtenção e baixo custo. Além disso, a medida da circunferência da cintura é um dos indicativos da presença de síndrome metabólica inclusive na adolescência(21). É importante ressaltar que por ser um estudo transversal, a relação de causalidade entre assistir TV em excesso e maior circunferência da cintura não pode ser estabelecida, porém a associação encontrada poderá subsidiar estudos longitudinais em países em desenvolvimento com características semelhantes ao Brasil, os quais possam esclarecer essa relação. Em países desenvolvidos, estudos longitudinais já mostram a relação de causalidade entre horas de TV e excesso de peso. Gortmaker et
Tabela 2. Análise bruta e ajustada entre assistir televisão mais do que 4 horas por dia e fatores biológicos, sociodemográficos, antropométricos e atividade física entre adolescentes, São Leopoldo (RS), Brasil, 2002-2003.

\begin{tabular}{|c|c|c|c|}
\hline & $\mathrm{Tv}>4 \mathrm{~h} / \mathrm{dia}$ & OR bruto & OR ajustado \\
\hline & $\%$ & (IC 95\%) & (IC 95\%) \\
\hline Meninos & 40,3 & 1 & 1 \\
\hline Meninas & 41,7 & $1,04(0,77-1,66)$ & $1,17(0,85-1,61)$ \\
\hline 16 a 19 anos de idade & 54,9 & 1 & 1 \\
\hline 10 a 15 anos de idade & 68,4 & $1,78(1,27-2,50)$ & $1,92(1,33-2,78)$ \\
\hline Mãe com até 8 anos de estudo & 53,4 & 1 & 1 \\
\hline Mãe com > 8 anos de estudo & 61,6 & $1,21(1,02-1,45)$ & $1,51(1,08-2,11)$ \\
\hline Tem irmãos & 51,2 & 1 & 1 \\
\hline Filho único & 61,3 & $1,50(1,01-2,23)$ & $1,38(0,91-2,08)$ \\
\hline Moram até 4 pessoas na casa & 58,5 & 1 & - \\
\hline Moram $>4$ pessoas na casa & 59,2 & $1,03(0,76-1,39)$ & \\
\hline Mãe trabalha fora & 58,2 & 1 & - \\
\hline Mãe não trabalha fora & 59,4 & $1,03(0,86-1,23)$ & \\
\hline Satisfeito com seu corpo & 57,0 & 1 & - \\
\hline Insatisfeito com seu corpo & 60,0 & $1,08(0,90-1,29)$ & \\
\hline CC $<$ percentil 80 & 47,8 & 1 & 1 \\
\hline$C C \geq$ percentil 80 & 61,3 & $1,35(1,11-1,64)$ & $1,63(1,09-2,44)$ \\
\hline Faz exercício físico regular & 58,0 & 1 & - \\
\hline Não faz exercício físico regular & 59,2 & $1,03(0,85-1,24)$ & \\
\hline
\end{tabular}

OR: Odds Ratio

IC: Intervalo de Confiança

CC: circunferência da cintura

al. ${ }^{(1)}$, por meio de um estudo de coorte, mostraram que assistir mais de cinco horas de TV por dia, em relação a assistir $\leq 2$ horas foi fator etiológico do desenvolvimento da obesidade e não conseqüência por inabilidade física ou isolamento social.

O comportamento de assistir TV em excesso na adolescência pode ser determinado, conforme demonstrado nesse estudo, pela maior autonomia do adolescente na faixa etária igual ou maior que 16 anos, que Ihe permite maior mobilidade social e menos tempo despendido dentro de casa. Essa associação entre menor faixa etária e maior tempo gasto assistindo TV também foi demonstrada por Eisenman et al. ${ }^{(9)}$ Estudos confirmaram que a inatividade física na infância transpõe para a adolescência ${ }^{(22)}$ e dessa para a vida adulta ${ }^{(23)}$. Sendo assim, quanto mais cedo 
houver intervenções para reduzir o sedentarismo menor a probabilidade do desenvolvimento de obesidade na adolescência e consequentemente menor risco para as doenças crônicas na vida adulta.

Foi demonstrado em outros estudos que o maior percentual de crianças e adolescentes que assistem TV em excesso estão nos estratos socioeconômicos menos privilegiados(24,25). Foi uma surpresa observar que mães com maior escolaridade está associado ao hábito excessivo de assistir TV, uma vez que seria de esperar que mães com mais tempo de educação formal fossem mais conscientes dos prejuízos dessa prática e limitassem a mesma. Essa associação foi demonstrada em alguns estudos ${ }^{(11,26,27)}$, porém, é importante ressaltar, que essas investigações foram realizadas em países desenvolvidos onde existe menor incidência de violência urbana quando comparada com a de países em desenvolvimento. Nesse contexto, uma hipótese que pode ser levantada é que mães de melhor escolaridade restringiriam seus filhos das atividades fora de casa com o receio que esses pudessem ficar mais vulneráveis à violência urbana. São necessárias outras investigações para confirmar a hipótese sugerida.

Estudo americano mostrou que adolescentes de áreas de maior criminalidade apresentaram maior freqüência de comportamento relacionado à inatividade física (horas com TV/vídeo/videogame/computador). O autor conclui que a atividade e inatividade física são influenciadas por fatores distintos. Enquanto que a atividade física é determinada por fatores ambientais (acesso à atividade física na escola, elevado índice de criminalidade na vizinhança), a inatividade física está associada com fatores sociodemográficos (escolaridade materna e renda familiar)(27). O hábito de assistir TV precocemente pode levar a criança a se tornar obesa e essa por sua vez agravar o sedentarismo pela capacidade limitada de fazer exercícios físicos e também pelo isolamento social que aumenta a chance daquele hábito ser a alternativa mais predominante de lazer. Portanto, os achados desse estudo devem ser utilizados para direcionar a elaboração de programas de saúde pública, especialmente por meio das escolas, enfocando adolescentes com faixa etária entre 10 e 15 anos, assim como devem servir de alerta para os profissionais de saúde e educadores.

\section{Limitações do estudo}

Por ser um estudo transversal, não é possível determinar relação de causa efeito, apenas de associação entre as variáveis, necessitando assim de estudos longitudinais, como discutido anteriormente. Por outro lado, estudos transversais são valiosos para a identificação de problemas e elaboração de hipóteses para os estudos longitudinais. Outra limitação se refere ao número consideravelmente superior de meninas que participaram do estudo. Por mais que os pais consintam com a participação de seus filhos, foram os próprios adolescentes que efetivamente consentiram ou não em participar. Nesse estudo, houve maior recusa por parte dos adolescentes do sexo masculino.

\section{CONCLUSÃO}

Nesse estudo, observou-se que é elevada a freqüência de adolescentes que assistem TV excessivamente e que esse comportamento está associado a adolescentes de menor faixa etária, com elevada adiposidade abdominal e com mães de maior escolaridade. Esses resultados justificam a elaboração de programas educativos em escolas para incentivar adolescentes a encontrarem outras alternativas de lazer.

Todos os autores declararam não haver qualquer potencial conflito de interesses referente a este artigo.

\section{REFERÊNCIAS BIBLIOGRÁFICAS}

1. Wang Y, Monteiro C, Popkin BM. Trends of obesity and underweight in older children and adolescents in the United States, Brazil, China and Rússia. Am J Clin Nutr. 2002; 75: 971-7.

2. Monteiro CA, Mondini L, Souza ALM, Popkin BM. The nutrition transition in Brazil. Eur J Clin Nutr 1995; 49: 105-13.

3. Monteiro CA, Mondini L, Costa RBL. Secular changes in dietary patterns in the metropolitan areas of Brazil (1988-1996). J Public Health 2000; 34: 251-8.

4. Troiano RP, Briefel RR, Carroll MD, Bialostosky K. Energy and fat intakes of children and adolescents in the United States: data from the national health and nutrition examination surveys. Am J Clin Nutr 2000; 72: 1343S-53S.

5. Crespo CJ, Smit E, Troiano RP, Bartlett SJ, Macera CA, Andersen RE. Television watching, energy intake, and obesity in US children: results from the Third National Health and Nutrition Examination Survey,1988- 1994. Arch Pediatr Adolesc Med 2001; 155: 360-5.

6. Giammattei J, Blix G, Marshak HH, Wollitzer AO, Pettitt DJ. Television watching and soft drink consumption: Associations with obesity in 11- to 13-year-old schoolchildren. Arch Pediatr Adolesc Med 2003; 157: 882-6.

7. Berkey CS, Rockett HRH, Field AE et al. Activity, dietary intake, and weight changes in a longitudinal study of preadolescent and adolescent boys and girls. Pediatrics 2000; 105: 1-9.

8. Dowda M, Ainsworth BE, Addy CL, Saunders R, Riner W. Environmental influences, physical activity, and weight status in 8- to 16-years-olds. Arch Pediatr Adolesc Med 2001; 155: 711-7.

9. Eisenmann JC, Bartee RT, Wang MQ. Physical Activity, TV Viewing, and Weight in U.S. Youth: 1999 Youth Risk Behavior Survey. Obes Res 2002; 10: 379-85

10. Janssen I, Katzmarzyk PT, Boyce WF, King MA, Pickett W. Overweight and obesity in canadian adolescents and their associations with dietary habits and physical activity patterns. J Adolesc Health 2004; 35: $360-7$.

11. Gortmaker SL, Must A, Sobol AM, Peterson K, Colditz GA, Dietz WH. Television viewing as a cause of increasing obesity among children in the United States, 1986 - 1990. Arch Pediatr Adolesc Med 1996; 150: 356-62.

12. American Academy of Pediatrics: Committee on Nutrition. Prevention of Pediatric Overweight and Obesity. Pediatrics 2003; 112: 424-30.

13. Utter J, Neumark-Sztainer D, Jeffery R, Story M. Couch potatoes or French fries: are sedentary behaviors associated with body mass index, physical activity, and dietary behaviors among adolescents? J Am Diet Assoc 2003; 103: 1298-305.
14. US Department of Health and Human Services. Healthy People 2010: Objectives for improving health (part B: focus areas 15-28). Washington DC, 2000.

15. Blair SN, Haskell WL, Ho P, Paffenbarger RS, Vranizan KM, Farquhar JW, et al. Assessment of habitual physical activity by a seven-day recall in a community survey and controlled experiments. Am J Epidemiol 1985; 122: 794-804.

16. Taylor RW, Jones IE, Williams SM, Goulding A. Evaluation of waist circumference, waist-to-hip ratio, and the conicity index as screening tools for high trunk fat mass, as measured by dual-energy $\mathrm{X}$-ray absorptiometry, in children aged 3-19 y. Am J Clin Nutr 2000; 72: 490-5.

17. American Academy of Pediatrics. Children, adolescents and television. Pediatrics. 2001; 107: 423-6.

18. Ribeiro IC, Taddei JA, Colugnatti F. Obesity among children attending elementary public schools in São Paulo, Brazil: a case-control study. Public Health Nutr 2003; 6: 659-63.

19. Monteiro P, Victora C, Barros F. Fatores de risco sociais, familiares e comportamentais para obesidade em adolescentes. Rev Panam Salud Publica 2004; 16: 250-8.

20. Hancox RJ, Milne BJ, Poulton R. Association of television viewing during childhood with poor educational achievement. Arch Pediatr Adolesc Med 2005; 159: 614-8.

21. Duncan GE, Li SM, Zhou XH. Prevalence and Trends of a Metabolic Syndrome Phenotype Among U.S. Adolescents, 1999-2000. Diabetes Care 2004; 27: 2438-43.

22. Blundell JE, King NA. Physical activity and regulation of food intake: current evidence. Med Sci Sports Exerc 1999; 31: S584-9.

23. Boynton-Jarret R, Thomas N, Peterson KE, Wiecha J, Sobol AM, Gortmaker SL. Impact of television viewing patterns on fruit and vegetable consumption among adolescents. Pediatrics 2003; 112: 1321-6.

24. Pate R, Trost S, Dowda M, Ott A, Ward D, Saunders R, Felton G. Tracking of physical activity, physical inactivity and health-related physical fitness in rural youth. Pediatr Exerc Sci 1999; 11: 364-76.

25. Raitakari OT, Porkka KV, Taimel S, Telama R, Rasanen L, Viikari JS, Effects of persistent physical activity and inactivity on coronary risk factors in children and young adults. The Cardiovascular Risk in Young Finns Study. Am J Epidemiol 1994; 140: 1905-205.

26. Hancox R, Milne B, Poulton R. Association between child and adolescent television viewing and adult health: a longitudinal birth cohort study. The Lancet 2004; 364: 257-62.

27. Gordon-Larsen P, McMurray RG, Popkin BM. Determinants of adolescent physical activity and inactivity patterns. Pediatrics 2000; 105: E83. 